\title{
The diagnostic value of the early extended fetal heart examination at 13 to 14 weeks gestational age in a high-risk population
}

\author{
Baoying Ye ${ }^{1,2,3 \#}$, Yi Wu ${ }^{4 \#}$, Jiong Chen ${ }^{1}$, Yu Yang ${ }^{1}$, Jianmei Niu $^{1}$, Hui Wang ${ }^{1}$, Yanlin Wang ${ }^{4}$, Weiwei Cheng ${ }^{2,3,5}$ \\ ${ }^{1}$ Department of Ultrasonography, the International Peace Maternity and Child Health Hospital, School of Medicine, Shanghai Jiao Tong University, \\ Shanghai, China; ${ }^{2}$ Shanghai Key Laboratory of Embryo Original Diseases, Shanghai, China; ${ }^{3}$ Shanghai Municipal Key Clinical Specialty, Shanghai, \\ China; ${ }^{4}$ Department of Prenatal Diagnostic Center, the International Peace Maternity and Child Health Hospital, School of Medicine, Shanghai Jiao \\ Tong University, Shanghai, China; ${ }^{5}$ Department of Obstetrics and Gynecology, the International Peace Maternity and Child Health Hospital, School \\ of Medicine, Shanghai Jiao Tong University, Shanghai, China \\ Contributions: (I) Conception and design: W Cheng, B Ye, Y Wu; (II) Administrative support: W Cheng, B Ye; (III) Provision of study materials \\ or patients: Y Wu, Y Wang; (IV) Collection and assembly of data: B Ye, Y Wu, J Chen, Y Yang, J Niu, H Wang, Y Wang; (V) Data analysis and \\ interpretation: B Ye, Y Wu, J Chen, Y Yang; (VI) Manuscript writing: All authors; (VII) Final approval of manuscript: All authors. \\ "These authors contributed equally to this work. \\ Correspondence to: Weiwei Cheng. Department of Obstetrics and Gynecology, the International Peace Maternity and Child Health Hospital, School \\ of Medicine, Shanghai Jiao Tong University, No. 910 Hengshan Road, Xuhui District, Shanghai 200030, China. Email: wwcheng29@shsmu.edu.cn.
}

Background: Congenital heart disease (CHD) is the most common congenital malformation that affects high-risk populations. A more definite heart diagnosis in the first trimester should be provided to guide clinical treatment. The study aim was to evaluate the diagnostic precision of the early extended fetal heart examination (EFHE) that includes abdominal situs view, four-chamber view (4CV), left ventricular outflow tract view (LVOT), right ventricular outflow tract view (RVOT), 3-vessel and tracheal view (3VT), ductal arch view, and the aortic arch view in the detection of CHD at the gestational age (GA) 13 to 14 weeks in a population with high risks.

Methods: This study was a diagnostic test study. EFHE was performed by transabdominal sonography in women at GA 13 to 14 weeks with singleton pregnancies who were at high risk for CHD. The risk of CHD was determined by family history of CHD, rubella infection, metabolic disorders, exposure to teratogens, conception by in-vitro fertilization (IVF), increased nuchal translucency (NT) thickness, abnormal obstetric ultrasound, etc. The operator had more than 5 years of experience in first-trimester scans and fetal echocardiography. Early scans were compared with a fetal echocardiography in the second trimester (16-24 weeks).

Results: EFHE was performed, and the pregnancy outcomes were obtained in 234 single pregnancies with a high risk of CHD. The average crown-rump length (CRL) was (76.17 \pm .09) mm. CHD was diagnosed in 43 cases by EFHE; 10 of these cases were misdiagnosed, and 2 cases were missed. Four cases were inconsistent in the main diagnosis of CHD. The sensitivity, specificity, positive predictive value (PPV), negative predictive value (NPV), and kappa coefficient (Ko) value of EFHE in diagnosing CHD by type and severity was $84.6 \%$ [95\% confidence interval (CI), 69.5-94.1\%], 96.9\% (95\% CI, 93.4-98.9\%), 84.6\% (95\% CI, 69.5-94.1\%), 96.9\% (95\% CI, 93.4-98.9\%), and 0.82 ( $\mathrm{P}<0.001)$, respectively.

Conclusions: EFHE can work as a diagnose examination for most major CHD cases at GA 13 to 14 weeks by an experienced operator; Therefore, this diagnostic system for fetal CHD may be applied in the first trimester. The study has been registered in the Chinese Clinical Trial Registry (www.chictr.org.cn). The registration number is ChiCTR2000038451.

^ ORCID: 0000-0003-0652-8810. 
Keywords: First trimester; congenital heart disease (CHD); high risk; echocardiography; early extended fetal heart examination (EFHE)

Submitted Jun 11, 2021. Accepted for publication Sep 22, 2021.

doi: $10.21037 / \mathrm{tp}-21-255$

View this article at: https://dx.doi.org/10.21037/tp-21-255

\section{Introduction}

Congenital heart disease (CHD), which occurs in 6.7-9.41 per 1,000 live births $(1,2)$, is the most common congenital malformation. Some characteristics can often help clinicians identify those patients at a high risk for CHDs in the first trimester. For example, a family history of CHD, rubella infection, metabolic disorders, exposure to teratogens, conception through in-vitro fertilization (IVF), increased nuchal translucency (NT) thickness, and abnormal obstetric ultrasound findings have been considered high risk for fetal CHD in the first trimester (3-5). Routine screenings for fetal heart anomalies are usually performed in the second trimester. However, this can lead to a delayed diagnosis of some types of CHDs with a poor prognosis, especially with chromosomal abnormalities. A pregnant woman has the right to decide whether to continue the pregnancy with a serious malformation, and have accurate information about the malformation as early as possible. Therefore, more definite heart diagnosis is needed for these high-risk populations in the first trimester.

Over the last decade, an increasing number of researchers had performed specialist fetal echocardiography in the first trimester of pregnancy, and their studies indicate that it is feasible to perform fetal echocardiography in the first trimester (6-8). However, most of these studies focused screening, not providing diagnostic tools. A fourchamber view (4CV) and 3-vessel and tracheal view (3VT) have been commonly used in these studies. The two views were not sufficient for a definite diagnosis; furthermore, some of these studies did not specify gestational age (GA) and some examinations were performed by the transvaginal transducers (6-8). Research indicates that the display rate of all the views that needed in the fetal cardiac examination increases by $20 \%$ in week $11,60 \%$ in week 12 , and $92 \%$ in week 13 , which suggests that the best time to perform fetal echocardiography in the first trimester is $13-14$ weeks of gestation (9).

With the development of ultrasonic instruments and probes, transabdominal transducers can image the fetal heart as clearly as transvaginal transducers, and can more flexibly obtain the ultrasonic views. Transabdominal examination is also more easily accepted by pregnant women.

Equipped with a high-resolution transabdominal transducer $(\mathrm{C} 2-9 \mathrm{MHz})$, the early extended fetal heart examination (EFHE) includes the abdominal situs view, $4 \mathrm{CV}$, left ventricular outflow tract view (LVOT), right ventricular outflow tract view (RVOT), 3VT, the ductal arch view, and the aortic arch view. The purpose of this study was to evaluate the diagnostic accuracy of EFHE, in the pregnancies identified to be with high risks of $\mathrm{CHD}$ at 13 to 14 weeks GA. We present the following article in accordance with the STARD reporting checklist (available at https://dx.doi.org/10.21037/tp-21-255).

\section{Methods}

\section{Study design}

Our diagnostic test study was conducted at the International Peace Maternity and Child Health Hospital (IPMCH) from December 2018 to October 2020. Pregnancies enrolled in the study were identified as being at a high risk for CHD from 13 to $14^{+6}$ weeks GA at IPMCH. According to the practice guideline (10), these patients had first trimester screening tests before the heart evaluation. The evaluation of the tricuspid regurgitation and reverse waves of the ductus venosus were not all included in this first-trimester screening. However, a color Doppler was used to evaluate $4 \mathrm{CV}$ of the heart. Patients who were enrolled signed an informed consent form. On the same day of the first scan, the height and weight of the pregnant women were measured, and then their maternal body mass index (BMI) was calculated in $\mathrm{kg} / \mathrm{m}^{2}$.

The study was conducted in accordance with the Declaration of Helsinki (as revised in 2013). The study was approved by the Institutional Ethics Committee of IPMCH (No.: GKLW 2019-24) and informed consent was taken from all the patients. The study has been registered with 
Table 1 Inclusion and exclusion criteria for this study

Inclusion criteria
Family history of CHD
Rubella infection
Metabolic disorders
Exposure to teratogens
Conception through IVF
Abnormal obstetrical ultrasound screen
Increased NT thickness ( $\geq 3$ mm)
Exclusion criteria
Multiple pregnancy
Without the second diagnosis results
Incomplete outcome information

$\mathrm{CHD}$, congenital heart disease; IVF, in-vitro fertilization; NT, nuchal translucency.

the Chinese Clinical Trial Registry (www.chictr.org.cn; no. ChiCTR2000038451).

\section{Inclusion and exclusion criteria}

Inclusion criteria was a GA in the range mentioned above and the presence any of the following high-risk factors for CHD (5): family history of CHD, rubella infection, metabolic disorders (e.g., diabetes), exposure to teratogens, conception through IVF, abnormal obstetric ultrasound screen (e.g., absence of nasal bone), increased NT thickness of $\geq 3.0 \mathrm{~mm}$, abnormal anatomy, etc. Cases missing results from a second diagnosis or lacking outcome information were not included in this study. A second diagnosis was determined by fetal echocardiography in the second trimester or before termination of pregnancy (TOP). Multiple pregnancies were not included in this study. The inclusion and exclusion flow-chart are shown in Table 1.

The following heart defects were excluded in CHD: mild valve regurgitation, atrial septal defect, right aortic arch, aberrant right subclavian artery, persistent left superior vena cava, cardiomyopathy, cardiac tumours and arrhythmias. Ventricular septal defect (VSD) was considered a mild cardiac CHD. Meanwhile, the following classified as major CHD: single ventricle (SV), persistent truncus arteriosus (PTA), double outlet right ventricle (DORV), transposition of the great arteries (TGA), tetralogy of Fallot (TOF), pulmonary atresia (PA), coarctation of the aorta (COA), interruption of aortic arch (IAA), hypoplastic left heart syndrome (HLHS), Ebstein anomaly, tricuspid valve dysplasia with severe regurgitation, atrioventricular septal defect (AVSD), and heterotaxia syndrome.

\section{Fetal beart examination}

The EFHE was performed by transabdominal ultrasound (Voluson $^{\mathrm{TM}}$ E10 GE ultrasound system with a 2-9 $\mathrm{MHz}$ sector probe; Zipf, Austria) and by an operator (Baoying $\mathrm{Ye}$ ) with more than 5 years of experience in first-trimester scans and fetal echocardiography. More than 2,000 cases of fetal echocardiography had been performed by the operator before this study.

The 7 views of each pregnancy included the abdominal situs view, 4CV, LVOT, RVOT, 3VT, the ductal arch view, and the aortic arch view. These heart views were visualized with two-dimensional (2D), color, and/or high-definition color flow Doppler (HD-Flow). HD-Flow is a power Doppler mode with direction. This protocol was named "EFHE". We used the cine loop to record the examinations and stored the digital video clips of the cardiac views in the ultrasound machines. Cases suspected of having CHD in the first trimester and then confirmed in the second trimester were classified as being diagnosed in the first trimester. If there were multiple CHDs in 1 case, only the main diagnosis was counted. If there were inconsistency in the 2 diagnoses, the scan in the first trimester was retrospectively reviewed.

The following scoring system was used to evaluate the quality of the images: 2 , the structures were clearly displayed; 1 , some structures were clearly displayed; and 0 , none of the structures were clearly displayed. According to the as low as reasonably achievable (ALARA) principle, the color Doppler application was performed as low as possible, and the total examining time was limited to 25 minutes.

All cases were re-assessed at least 3 weeks later in the second trimester or before TOP by another experienced fetal cardiologist.

\section{Cytogenetic and molecular testing}

If CHDs or other abnormalities (including NT $\geq 3.0 \mathrm{~mm}$ and abnormalities of system structure) were identified, the karyotype analysis and chromosomal microarray analysis (CMA) by chorionic villus sampling (CVS) were recommended before 14 weeks, or amniocentesis was recommended for late GA. 
Table 2 The basic characteristics of the 234 cases

\begin{tabular}{lcc}
\hline Characteristics & Mean \pm SD & Range \\
\hline Maternal age (years) & $32.58 \pm 5.17$ & 19 to 52 \\
Gravidity & $2.16 \pm 1.33$ & 1 to 8 \\
Parity & $0.38 \pm 0.53$ & 0 to 2 \\
Maternal BMI & $21.5 \pm 2.76$ & 14.4 to 30.6 \\
Fetal CRL & $76.17 \pm 7.09$ & 65 to 90
\end{tabular}

$\mathrm{SD}$, standard deviation; BMI, body mass index; CRL, crownrump length.

Table 3 Indications for EFHE in the first trimester

\begin{tabular}{lc}
\hline High risk & $\mathrm{N}(\%)$ \\
\hline Increased NT thickness $(\geq 3 \mathrm{~mm})$ & $116(49.6)$ \\
Metabolic disease & $10(4.3)$ \\
Conception through IVF & $40(17.1)$ \\
Rubella infection & $20(8.5)$ \\
Exposure to teratogens & $12(5.1)$ \\
Previous pregnancy with CHD & $20(8.5)$ \\
Abnormal obstetrical ultrasound screen & $16(6.8)$
\end{tabular}

EFHE, early extended fetal heart examination; NT, nuchal translucency; IVF, in-vitro fertilization; CHD, congenital heart disease.

\section{Data collection/follow-up}

In cases of CHD, a multidisciplinary team counsel was then provided to manage the prognosis further. The multidisciplinary team usually included an obstetrician, a geneticist, a fetal medicine expert, a neonatologist and a pediatric cardiac surgeon.

The postnatal outcome was obtained from the hospital record of the new-born or directly from the parents. In CHD cases, a neonatal echocardiography was performed and recorded before hospital discharge by another examiner (about 2 to 4 days after delivery). Autopsy reports were obtained if performed in stillborn or termination cases. Clinical examinations by an experienced pediatrician were performed on all infants, including auscultation of heart murmurs and oxygen saturation examination of the upper and lower extremities. If no anomaly was suspected or detected, the neonate/fetus was considered normal.

\section{Sample size}

According to the literature and our previous clinical experience (11), the sensitivity and specificity were 0.8 and 0.9 respectively. The prevalence of CHD in the high-risk group was about $10 \%$ (12). The sample size was estimated using PASS 15.0 software (National Council for the Social Studies, Kaysville, UT, USA), with $\alpha=0.05$ and $\beta=0.1$. At least 230 cases were included, including 23 fetuses with CHD. After a $15 \%$ loss-to-follow-up rate, 271 cases were included in the study $(\mathrm{n}=271)$.

\section{Statistical analysis}

Statistical software was used by SPSS 25.0 (IBM Corporation, Armonk, NY, USA). Numerical data were represented as mean \pm standard deviation $(\mathrm{SD})$. The chisquare test was used to compare chromosome abnormality rates in cases with and without abnormal EFHE. The significance level was 0.05 (2-tailed). The rate and the $95 \%$ confidence interval (CI) of sensitivity, specificity, positive predictive value (PPV), and negative predictive value (NPV) were calculated for EFHE to detect any CHD at the 13 to 14 weak scans. The kappa coefficient (Ko) was calculated to assess the consistency between the EFHE and the second diagnosis. Agreement was classified according to Ko values, including almost perfect (81-100\%), substantial (61-80\%), moderate (41-60\%), fair (21-40\%), slight (1-20\%), and poor $(\leq 0)(13)$.

\section{Results}

During the study period, 271 women with singleton pregnancy received EFHE during the study period. 37 were excluded: 8 were lost to follow-up, and 29 were TOP or died without a secondary diagnosis. Ultimately, 234 cases were enrolled. The basic characteristics of the 234 cases are shown in Table 2. The median maternal age and BMI were 32.58 (range, 19-52) years and 21.5 (range, 14.4-30.6) $\mathrm{kg} / \mathrm{m}^{2}$, respectively. The median fetal crown-rump length (CRL) was 76.17 (range, 65-90) mm. Indications for ultrasound are shown in Table 3. Most (49.6\%) of the fetuses were recommended for EFHE with increased NT. A transabdominal scan was performed in all the cases.

The results of the first and second diagnoses are shown in Figure 1. The incidence of CHD in this study was $16.7 \%$ (39/234) for the high-risk population, and $33(14.1 \%)$ cases 


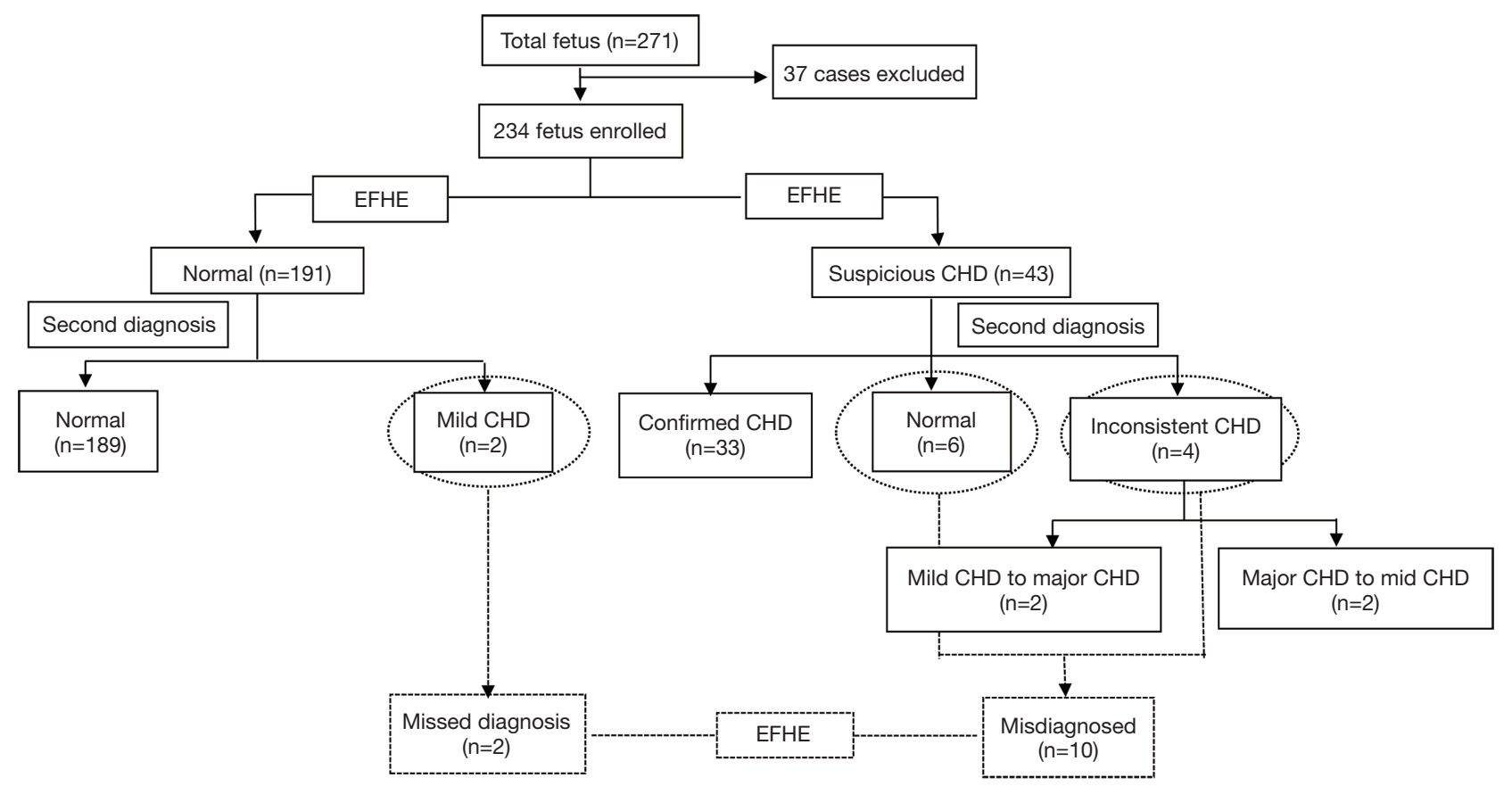

Figure 1 Flowchart showing the protocol and results of the 2 diagnoses for pregnancies with high risks of CHD. EFHE, early extended fetal heart examination; CHD, congenital heart disease.

of CHD were both diagnosed on the 2 scans $(33 / 234)$. With EFHE, there were 43 abnormal fetuses, with 10 cases misdiagnosed and 2 cases missed on the first scan. The main diagnosis of CHD was inconsistent in 4 cases between the 2 diagnoses. Compared to the fetal echocardiography in the second trimester, the performance of EFHE in detecting any CHD showed a sensitivity of $94.9 \%$ (95\% CI, 82.7-99.4\%), a specificity of $96.9 \%$ (95\% CI, 93.4-98.9\%), a PPV of $86.0 \%$ (95\% CI, 72.1-94.7\%) and an NPV of 99.0\% (95\% CI, 96.3-99.9\%). There was almost perfect consistency between EFHE and the second diagnosis (Ko $=0.88, \mathrm{P}<0.001)$. In terms of the diagnostic consistency of the type and severity of CHD, EFHE showed a sensitivity of $84.6 \%$ (95\% CI, 69.5-94.1\%), a specificity of $96.9 \%(95 \%$ CI, 93.4-98.9\%), a PPV of 84.6\% (95\% CI, 69.5-94.1\%), and an NPV of $96.9 \%$ (95\% CI, 93.4-98.9\%). The Ko value was $0.82(\mathrm{P}<0.001)$. Table 4 shows the indication for referral, extracardiac malformation, karyotype, CMA results, and outcomes in all cases of CHD or suspected CHD. Among the CHD group, 19 cases (48.7\%) had extracardiac malformations, and $86.0 \%$ (37/43) of fetuses with CHD that were diagnosed in the first trimester were terminated. Most of them had major CHDs. The detection rate of EFHE for the different types of CHD are shown in Table 5.
The detection rate of EFHE was high in the diagnosis of most CHDs, especially in major ones. However, it was poor for VSD (20.0\%). In this study, the most common lesions were TOF ( 8 cases, $20.5 \%$ ) and SV (6 cases, $15.4 \%)$. The quality of the images of normal fetuses in the first trimester is summarized in Table 6 and indicates that most views needed in the EFHE could be obtained.

Karyotyping and CMA were performed in 155 cases, of whom 44 cases had abnormal chromosome karyotypes or positive CMA. Chromosomal abnormalities were found in 23 of the $32(71.9 \%)$ cases with cardiac abnormalities and 21 of the $123(17.1 \%)$ cases with a normal heart, $\left(\chi^{2}=37.515, \mathrm{P}<0.001\right)$. Figure 2 shows the types of CHD diagnosed at 13-14 weeks and their frequencies in different fetal karyotypes (10 cases normal, 8 cases trisomy 21 (T21), 6 cases trisomy 18 (T18), 3 cases trisomy 13 (T13), 2 cases Turner syndrome, and 3 cases with other abnormal). Noninvasive prenatal testing (NIPT) was chosen in 56 cases with negative EFHE and without other malformation to exclude common aneuploidies, presenting low-risk results. In all, 23 participants declined any chromosomal or genetic tests, and 8 of whom terminated pregnancy due to severe phenotypes of CHD or extracardiac abnormalities, and 15 of whom had normal births with normal clinical examination. 
Table 4 Indication for referral, antenatal cardiac finding, extracardiac malformation, karyotype, CMA results, and outcome in all cases of CHD and suspected CHD

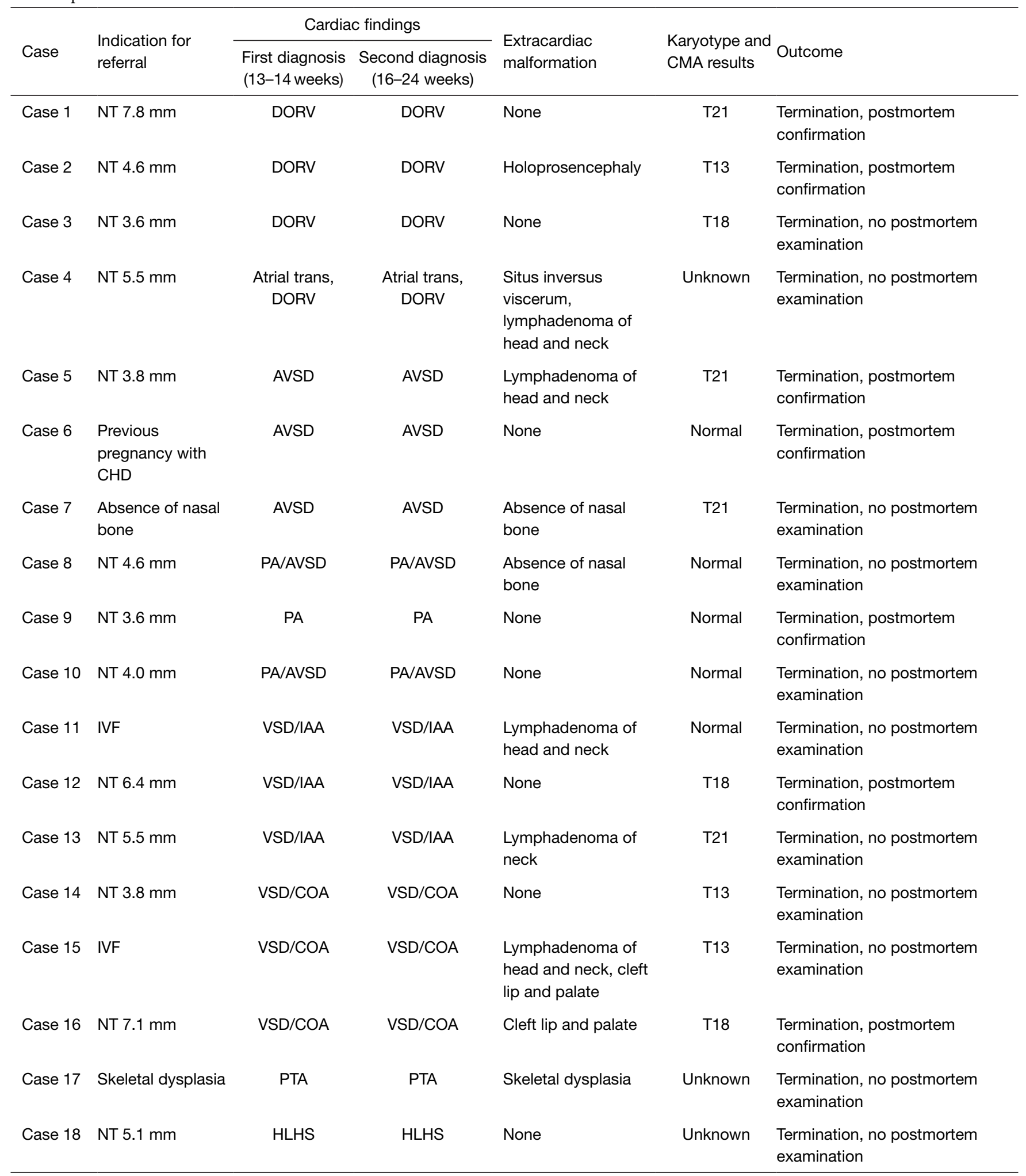

Table 4 (continued) 
Table 4 (continued)

\begin{tabular}{|c|c|c|c|c|c|c|}
\hline Case & $\begin{array}{l}\text { Indication for } \\
\text { referral }\end{array}$ & \multicolumn{2}{|c|}{ Cardiac findings } & $\begin{array}{l}\text { Extracardiac } \\
\text { malformation }\end{array}$ & $\begin{array}{l}\text { Karyotype and } \\
\text { CMA results }\end{array}$ & Outcome \\
\hline Case 20 & IVF & SV & SV & None & Unknown & $\begin{array}{l}\text { Termination, no postmortem } \\
\text { examination }\end{array}$ \\
\hline Case 21 & NT $5.3 \mathrm{~mm}$ & SV & SV & $\begin{array}{l}\text { Omphalocele, cyst } \\
\text { of cord }\end{array}$ & $\mathrm{T} 18$ & $\begin{array}{l}\text { Termination, no postmortem } \\
\text { examination }\end{array}$ \\
\hline Case 22 & $\begin{array}{l}\text { Previous } \\
\text { pregnancy with } \\
\mathrm{CHD}\end{array}$ & SV & SV & Exencephaly & Unknown & $\begin{array}{l}\text { Termination, no postmortem } \\
\text { examination }\end{array}$ \\
\hline Case 25 & Pleural effusion & TGA & TGA & $\begin{array}{l}\text { Absence of nasal } \\
\text { bone }\end{array}$ & unknown & $\begin{array}{l}\text { Termination, no postmortem } \\
\text { examination }\end{array}$ \\
\hline Case 26 & NT $5.6 \mathrm{~mm}$ & TOF & TOF & None & $\mathrm{T} 21$ & $\begin{array}{l}\text { Termination, postmortem } \\
\text { confirmation }\end{array}$ \\
\hline Case 27 & NT $3.4 \mathrm{~mm}$ & TOF & TOF & None & $\mathrm{T} 21$ & $\begin{array}{l}\text { Termination, postmortem } \\
\text { confirmation }\end{array}$ \\
\hline Case 28 & NT $3.5 \mathrm{~mm}$ & TOF & TOF & $\begin{array}{l}\text { Lymphadenoma of } \\
\text { head and neck }\end{array}$ & $\mathrm{T} 21$ & $\begin{array}{l}\text { Termination, no postmortem } \\
\text { examination }\end{array}$ \\
\hline Case 32 & $\begin{array}{l}\text { Previous } \\
\text { pregnancy with } \\
\mathrm{CHD}\end{array}$ & TOF & TOF & None & $\begin{array}{c}1 \mathrm{p} 36 \\
\text { microdeletion }\end{array}$ & $\begin{array}{l}\text { Termination, no postmortem } \\
\text { examination }\end{array}$ \\
\hline Case 33 & IVF & Possible TOF & VSD & None & NIPT normal & LB \\
\hline Case 34 & IVF & Normal & VSD & None & Normal & LB \\
\hline Case 35 & $\begin{array}{l}\text { Exposure to } \\
\text { teratogens }\end{array}$ & VSD & VSD & None & $\begin{array}{c}5 q 14 \\
\text { microdeletion }\end{array}$ & $\begin{array}{l}\text { Termination, no postmortem } \\
\text { examination }\end{array}$ \\
\hline Case 36 & $\begin{array}{l}\text { Lymphadenoma } \\
\text { of head and neck }\end{array}$ & VSD & TOF & $\begin{array}{l}\text { Lymphadenoma of } \\
\text { head and neck }\end{array}$ & Normal & $\begin{array}{l}\text { Termination, no postmortem } \\
\text { examination }\end{array}$ \\
\hline Case 37 & Rubella infection & VSD & VSD/COA & None & Normal & LB \\
\hline Case 38 & $\begin{array}{l}\text { Exposure to } \\
\text { teratogens }\end{array}$ & Possible TOF & VSD & Holoprosencephaly & $\mathrm{T} 13$ & $\begin{array}{l}\text { Termination, postmortem } \\
\text { confirmation }\end{array}$ \\
\hline
\end{tabular}

Table 4 (continued) 
Table 4 (continued)

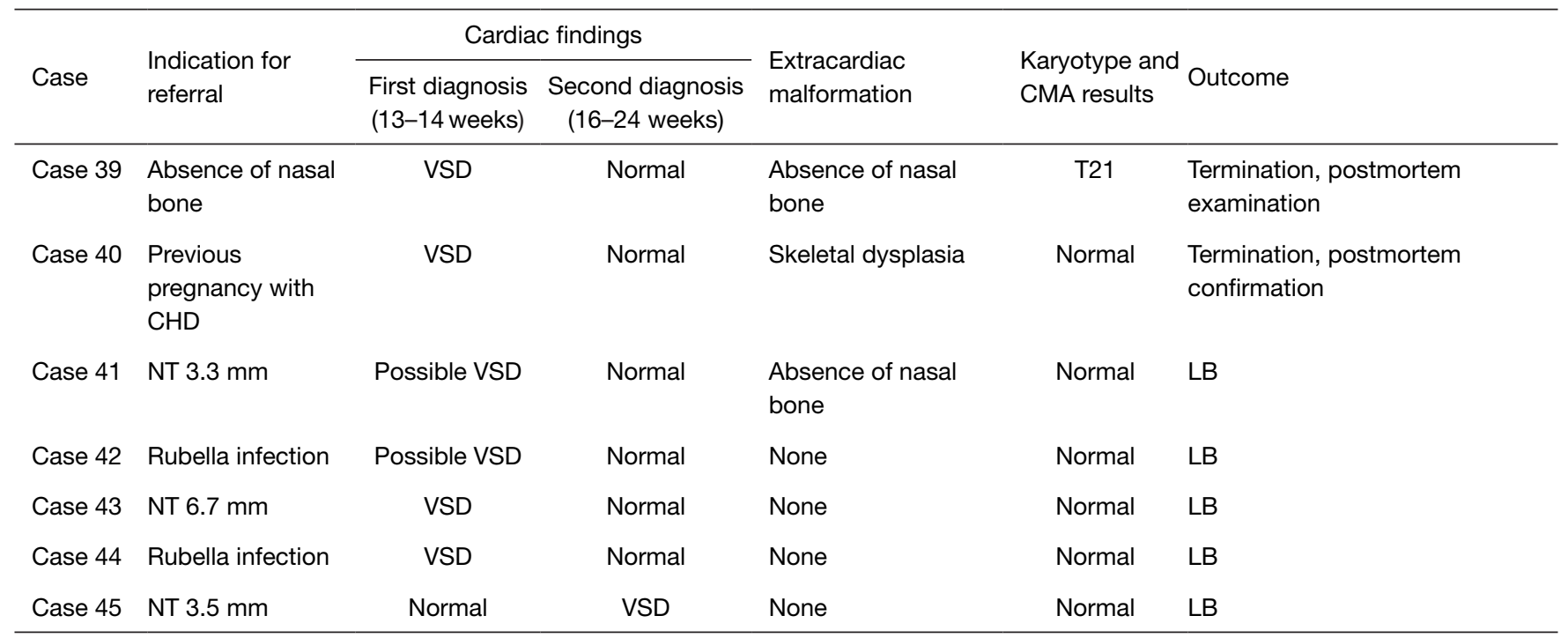

CMA, chromosomal microarray analysis; CHD, congenital heart disease; NT, nuchal translucency; IVF, in-vitro fertilization; DORV, double outlet right ventricle; AVSD, atrioventricular septal defect; PA, pulmonary atresia; HLHS, hypoplastic left heart syndrome; VSD, ventricular septal defect; IAA, interruption of aortic arch; COA, coarctation of the aorta; PTA, persistent truncus arteriosus; SV, single ventricle; TGA, transposition of the great arteries; TOF, tetralogy of Fallot; T21, trisomy 21; T13, trisomy 13; T18, trisomy 18; NIPT, non-invasive prenatal testing; LB, live birth at term.

Table 5 The detection rate of EFHE in different types of CHD

\begin{tabular}{|c|c|c|c|c|c|c|}
\hline Cardiac abnormality & \multicolumn{3}{|c|}{ Second diagnosis $(n=39)$} & \multicolumn{3}{|c|}{ EFHE $(n=43)$} \\
\hline TOF & 8 & 20.5 & $9.3-36.5$ & 7 & 2 & 87.5 \\
\hline SV & 6 & 15.4 & $5.9-30.5$ & 6 & 0 & 100.0 \\
\hline VSD & 5 & 12.8 & $4.3-27.4$ & 1 & 5 & 20.0 \\
\hline $\mathrm{COA}$ & 4 & 10.3 & $2.9-24.2$ & 3 & 1 & 75.0 \\
\hline AVSD & 3 & 7.7 & $1.6-20.9$ & 3 & 0 & 100.0 \\
\hline PA & 3 & 7.7 & $1.6-20.9$ & 3 & 0 & 100.0 \\
\hline IAA & 3 & 7.7 & 1.6-20.9 & 3 & 0 & 100.0 \\
\hline TGA & 1 & 2.6 & $0.1-13.5$ & 1 & 0 & 100.0 \\
\hline Total & 39 & - & - & 35 & 8 & 89.7 \\
\hline
\end{tabular}

EFHE, early extended fetal heart examination; CHD, congenital heart disease; Cl, confidence interval; TOF, tetralogy of Fallot; SV, single ventricle; VSD, ventricular septal defect; DORV, double outlet right ventricle; COA, coarctation of the aorta; AVSD, atrioventricular septal defect; PA, pulmonary atresia; IAA, interruption of aortic arch; HLHS, hypoplastic left heart syndrome; PTA, persistent truncus arteriosus; TGA, transposition of the great arteries. 


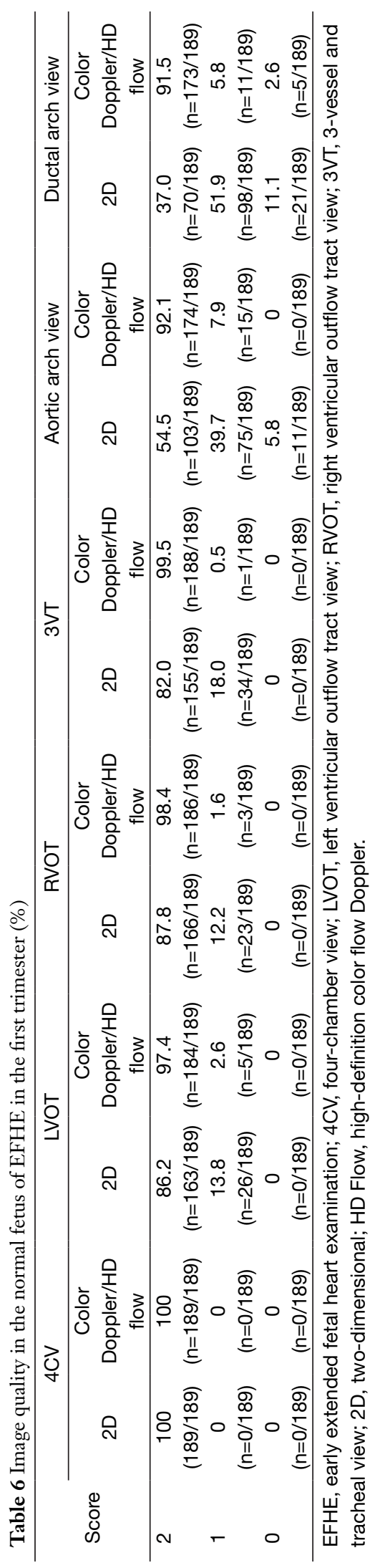

\section{Inconsistent and misdiagnosis case analysis}

The main diagnosis in these 12 cases was inconsistent between the 2 scans. One case of VSD detected in the first trimester was diagnosed as TOF on the second scan. Two cases suspected of TOF in the first trimester were diagnosed as only VSD in the second trimester. A case of VSD was later diagnosed as VSD with COA on the second scan. Six cases were detected as VSD (diameter of defects: $0.8-1.2 \mathrm{~mm}$ ) at the first scan, but then had no abnormal findings in the second trimester, and were normal in the neonatal period. Two cases of VSD in 24 weeks were missed on the first scan.

\section{Discussion}

This study recommends a novel clinical ultrasound examination protocol, EFHE, to diagnose CHD in the high-risk population at GA 13-14 weeks. The findings of this study demonstrate that the EFHE can be carried out at 13 to 14 weeks, with an NPV (96.9\%). We found that more than $95 \%$ of major CHDs can be diagnosed during this GA. There was perfect consistency between EFHE and the second diagnosis $(\mathrm{Ko}=0.82, \mathrm{P}<0.001)$. Our findings are consistent with those of $\mathrm{Yu}$ et al. (11) who collected data from 26,201 fetal hearts in the review of 18 studies at this stage. They found the sensitivity and specificity were $75 \%$ and $99.9 \%$, respectively. Thus, the sensitivity of our study was reliable for the diagnosis of most major CHDs.

It should be noted that the experience of operators can affect the detection rate of heart disease (14). Tegnander et al. (15) reported that sonographers with experience of fetal echocardiography more than 2,000 cases had a higher detection rate of CHD as compared to the operators performing fewer than 2,000 cases $(52 \%$ vs. $32.5 \%)$. So, the operator was an expert with more than 5 years of experience specialized in first-trimester scans and fetal echocardiography, and more than 2,000 cases of fetal echocardiography had been performed by the operator before this study.

This protocol didn't scan the pulmonary vein, superior vena cava or inferior vena cava. Hutchinson et al. (16) reported that the pulmonary vein could only be observed in $42 \%$ of cases between 13 and 14 weeks. Additionally, only abnormalities of the vena cava reflux were not complex cardiac malformations. Thus, referring to the requirements for echocardiography in the second trimester (5) and previous research results $(6,8,16)$, we proposed this detailed examination protocol for the first trimester heart 


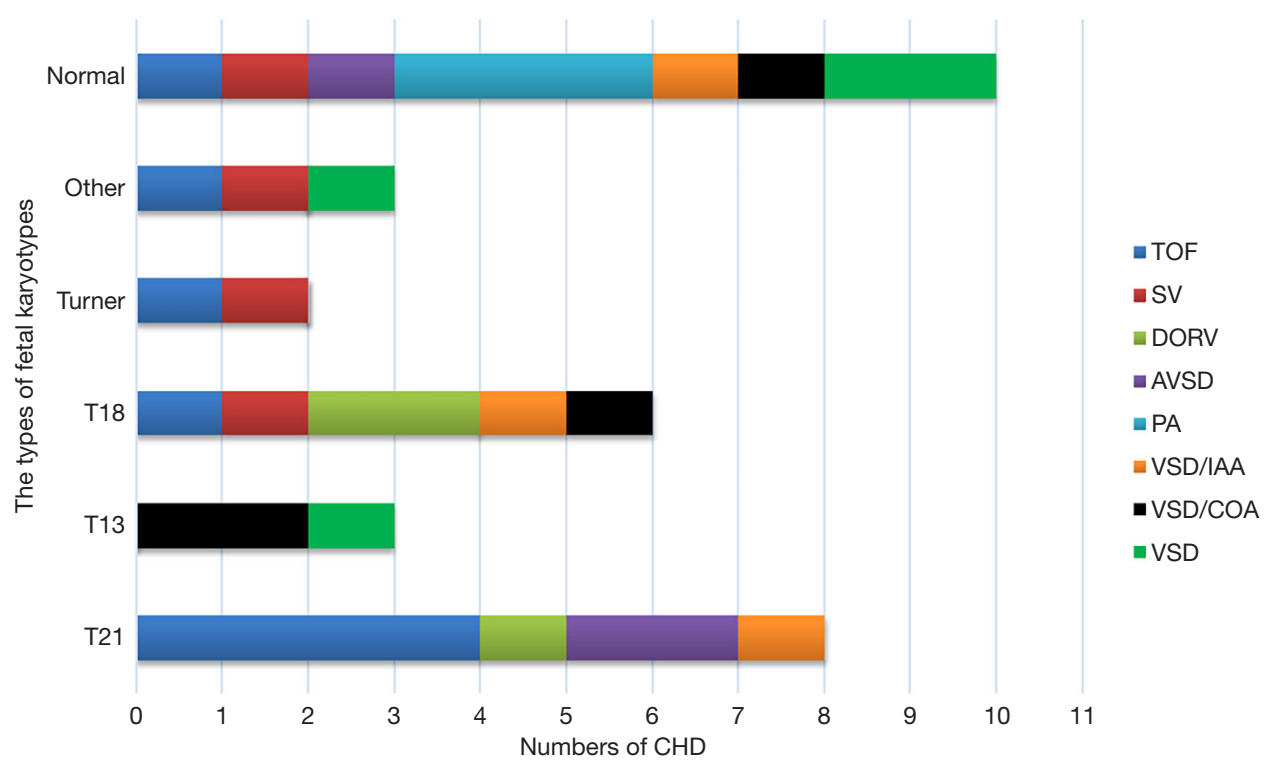

Figure 2 Graph showing the number of CHD fetuses in different karyotypes. CHD, congenital heart disease; TOF, tetralogy of Fallot; SV, single ventricle; DORV, double outlet right ventricle; AVSD, atrioventricular septal defect; PA, pulmonary atresia; VSD, ventricular septal defect; IAA, interruption of aortic arch; COA, coarctation of the aorta.

examination, and we chose to examine at 13 to 14 weeks of gestation. This is also a suitable period for a villous puncture (17). In common, the fetal echocardiography is usually done after 18 weeks of gestation. Waiting until 18 weeks GA causes the parents to become anxious if the fetus is identified as high risk for CHD or a chromosomal anomaly. Therefore, EFHE is performed at 13 to 14 weeks GA in fetuses as being at high risk for CHD. This allows for molecular diagnostics to recommended sooner when a heart abnormality is found, as results will be available in 3 to 4 weeks. Early pregnancy interventions can be performed for some patients with a poor prognosis of the CHD, at least 4 weeks in advance. The earlier the TOP for fetal malformation was performed, the better the psychological recovery $(18,19)$. If patients choose to continue this pregnancy, they can benefit more from this additional time from early diagnosis, which allows them to prepare and plan for their fetus with further health supervision, including choosing to deliver in a reference center, arranging antenatal care, and making an appointment for a neonatal cardiac surgeon. Meanwhile, a normal diagnosis can lower anxiety in high-risk pregnant women.

We documented that the cardiac malformations had a higher incidence in the high-risk population of $16.7 \%$, which is similar to the $14.4 \%$ reported in Gabriel's study (12). Also, chromosomal abnormalities were more common among this population. In our series, at least $71.9 \%$ of CHDs diagnosed in the first trimester were associated with abnormal chromosome results, which is significantly higher than the rate for abnormalities detected with a normal heart. The frequency of chromosomal abnormalities in fetuses was higher than that reported in previous research (11), which did not specifically select high-risk pregnancies. Furthermore, $86.0 \%$ of fetuses with CHDs diagnosed in the first trimester were terminated, consistent with the figures reported by Jicinska et al. (20). Early diagnosis of severe types of CHDs with or without chromosomal abnormalities leads to increased pregnancy termination rates. In our study, the most common lesions were TOF in 8 cases (20.5\%), SV in 6 cases (15.4\%), VSD in 5 cases $(12.8 \%)$, DORV in 4 cases $(10.3 \%)$ and COA in 4 cases $(10.3 \%)$. This result was different from that of Karadzov et al.'s study (21), in which HLHS (27.4\%) and TOF $(19.3 \%)$ were most common conditions. This may be due to the differences in the race of the study participants. In our study, T21 was the most common chromosomal abnormality in TOF, that was maybe due to the small sample. But there indeed may exist a different distribution of chromosomal abnormalities in early pregnancy, especially in a high-risk population.

Many studies $(22,23)$ focused on CHD scanning in the first trimester, and most of them did not select a particular 

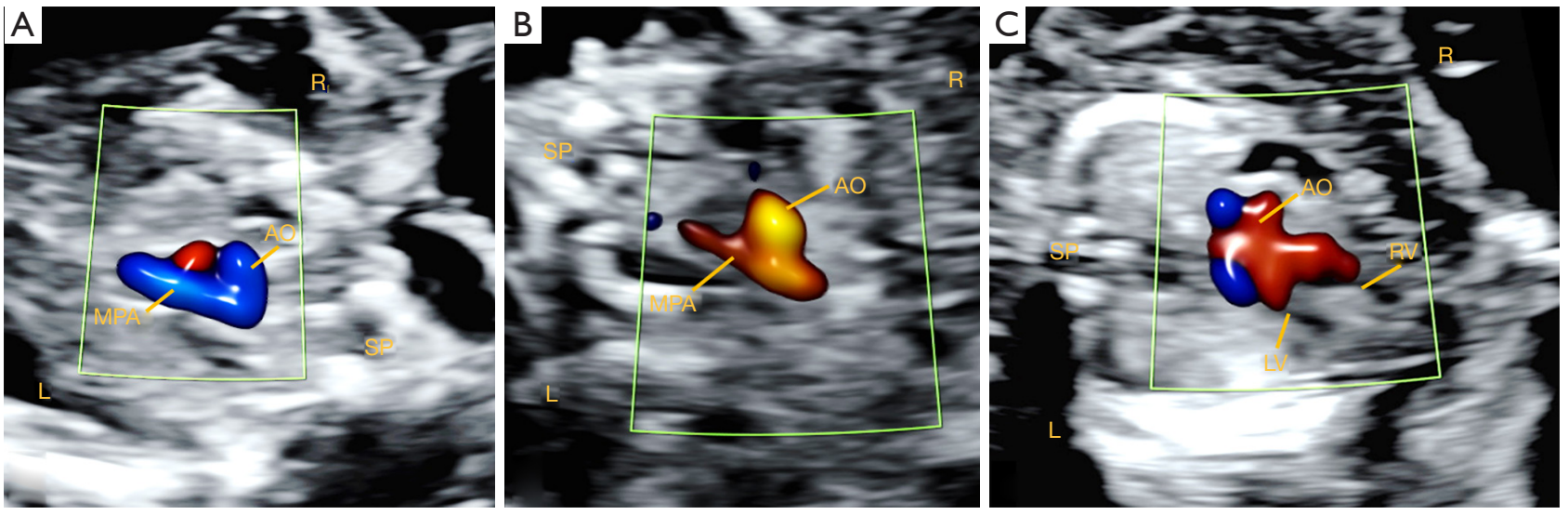

Figure 3 Ultrasound images show pulmonary stenosis and the dilated aortic artery in the second trimester. (A) Ultrasound image showing no obvious pulmonary stenosis at $13^{+4}$ weeks of gestation. (B) The severe hypoplasia of the MPA at $16^{+3}$ weeks of gestation. (C) Enlarged AO overriding the ventricular septum at $16^{+3}$ weeks of gestation. MPA, main pulmonary artery; AO, aorta; SP, spine; L, left; R, right; LV, left ventricle; RV, right ventricle.

GA. However, Haak et al. (9) suggested that more details can be viewed later in the first trimester. Thus, we selected 13 to 14 weeks of GA for the EFHE. To our knowledge, this is the first study to explicitly outline the protocols and GA for the fetal echocardiography in the first trimester. One of the reasons for the higher success rate in the present study is the progress of probe technology and ultrasonic instruments. In this study, a $2-9 \mathrm{MHz}$ transabdominal sector probe was used for scanning. Higher frequencies of transabdominal probes can show more detailed information of the small structures in the first trimester. Transabdominal probes can obtain the views of the heart more flexibly and easily. The high quality of color-flow mapping and extensive experience of the operators are also conducive to a high detection rate of CHDs. Visualization rates demonstrate that 4-CV, LVOT/RVOT and 3VT, aortic arch view, and ductal arch view were displayed in almost every patient, which is in line with findings of the literature (24). Most major CHDs can be diagnosed at this stage, not merely screened.

Although most complex CHDs can be detected in the first trimester, early diagnosis of some types of CHD remains a challenge. One case of TOF was not diagnosed at 13 weeks GA, but it was diagnosed at 16 weeks GA before termination. A retrospective review of the recorded loops obtained in the first trimester showed that an outlet VSD could be found, but hypoplasia of the pulmonary artery and the sign of aorta (AO) that override the ventricular septum were not very obvious. At 16 weeks, ultrasound images showed severe hypoplasia of the main pulmonary artery (MPA) and an enlarged aortic artery overriding the ventricular septum (Figure 3). Some studies also reported this misdiagnosis $(25,26)$. The outlet VSD with anterior malalignment can progress to TOF in the late GA. Wu et al. (27) observed that 4CV was generally normal in fetal TOF, and pulmonary stenosis was not always obvious with a GA of less than 24 weeks. They proposed that AO dilatation and the abnormity ratio of MPA to AO artery (MPA/AO) are markers in the diagnosis of TOF in fetus. In another 2 cases of VSD diagnosed as TOF in the first scan, mild aortic override and pulmonary artery stenosis could be observed in a retrospective review of the recorded material. Thus, the diagnostic accuracy of TOF in the first trimester presents challenges.

We can describe the VSD and MPA/AO ratio instead of a TOF diagnosis in the atypical cases, and then a reexamining of the ultrasound will can be completed in a later GA. One case of VSD with COA was diagnosed as VSD only. AO stenosis was mild in the second trimester. The recorded materials were retrospectively analysed, which showed that the AO was not significantly smaller than the pulmonary artery in the first trimester. These types of missed diagnosis of the CHD may be due to the natural development of the disease. Six cases were diagnosed with VSD at the first trimester, with no abnormal evident in the second trimester and neonatal period. The reason for this may be morphological progression, as small VSDs can undergo spontaneous closure. Thus, 
these cases may represent the real progression of the heart during pregnancy, rather than a misdiagnosis. However, the ultrasonic artefacts may still be the reason for the misdiagnosis. Therefore, in all continuing pregnancies, the first trimester scan should always be followed by fetal echocardiography in the second trimester to confirm normality. Abnormal findings detected at 13-14 weeks scan should be reassessed to detect any cardiac malformation missed in the first trimester. Second-trimester scans would provide better assessment of the heart structures, like the pulmonary venous connections, the interventricular septum and so on.

However, the potential risks of ultrasound should be carefully considered, and the ALARA principle (28) should be followed in the first trimester scan. The newer generation of ultrasound equipment is recommended, as it may provide higher safety indices in the heart examination of the first-trimester (29). Indeed, only the most up-to-date technology should be used for conducting first-trimester echocardiography. Otherwise, EFHE should be performed late in the first trimester, which will be safer for the fetus.

\section{Limitations of the study}

This study has some limitations which should be noted. First, not all autopsies are available in cases of fetal death or in which patients choose to terminate the pregnancy with aneuploidy or serious fetal defect. However, all enrolled cases were re-assessed at least 3 weeks later in the second trimester by another experienced fetal cardiologist. Secondly, echocardiography is not performed in all infants, but only in those diagnosed with CHD during pregnancy. However, a clinical examination was performed for every infant by an experienced pediatrician and included the auscultation of heart murmurs and the oxygen saturation examination of the upper and lower extremities. Finally, the study cannot be used to evaluate the diagnostic performance of EFHE in a low-risk population. The high prevalence of CHD in the study may be related to the satisfactory performance of EFHE; nonetheless, our study demonstrates that an ultrasound examination protocol conducted at 13-14 weeks GA by a specialist fetal cardiologist in a highrisk population can achieve high precision.

\section{Conclusions}

EFHE conducted by an experienced operator could diagnose most major CHD cases at 13-14 weeks GA, and may provide better opportunities for understanding the evolution of CHDs early from the first trimester. Chromosome abnormalities were more common among CHD cases. The diagnostic system for fetal CHD may be applied in the first trimester, especially in the high-risk population.

\section{Acknowledgments}

The authors would like to thank all the fetus and parents who participated in the study.

Funding: This work was supported by the Shanghai Committee of Science and Technology, China (Grant number 18411963500 and 19411960900$)$.

\section{Footnote}

Reporting Checklist: The authors have completed the STARD reporting checklist. Available at https://dx.doi. org/10.21037/tp-21-255

Data Sharing Statement: Available at https://dx.doi. org/10.21037/tp-21-255

Conflicts of Interest: All authors have completed the ICMJE uniform disclosure form (available at https://dx.doi. org/10.21037/tp-21-255). The authors have no conflicts of interest to declare.

Ethical Statement: The authors are accountable for all aspects of the work in ensuring that questions related to the accuracy or integrity of any part of the work are appropriately investigated and resolved. The study was conducted in accordance with the Declaration of Helsinki (as revised in 2013). The study was approved by the Institutional Ethics Committee of IPMCH (No.: GKLW 2019-24) and informed consent was taken from all the patients.

Open Access Statement: This is an Open Access article distributed in accordance with the Creative Commons Attribution-NonCommercial-NoDerivs 4.0 International License (CC BY-NC-ND 4.0), which permits the noncommercial replication and distribution of the article with the strict proviso that no changes or edits are made and the original work is properly cited (including links to both the formal publication through the relevant DOI and the license). See: https://creativecommons.org/licenses/by-nc-nd/4.0/. 


\section{References}

1. Mat Bah MN, Sapian MH, Jamil MT, et al. The birth prevalence, severity, and temporal trends of congenital heart disease in the middle-income country: A populationbased study. Congenit Heart Dis 2018;13:1012-27.

2. Liu Y, Chen S, Zühlke L, et al. Global birth prevalence of congenital heart defects 1970-2017: updated systematic review and meta-analysis of 260 studies. Int J Epidemiol 2019;48:455-63.

3. Ozyuncu O, Tanacan A, Fadiloglu E, et al. Impact of Increased Nuchal Translucency Values on Pregnancy Outcomes: A Tertiary Center Experience. Fetal Pediatr Pathol 2021;40:189-97.

4. Minnella GP, Crupano FM, Syngelaki A, et al. Diagnosis of major heart defects by routine first-trimester ultrasound examination: association with increased nuchal translucency, tricuspid regurgitation and abnormal flow in ductus venosus. Ultrasound Obstet Gynecol 2020;5 5:637-44.

5. American Institute of Ultrasound in Medicine. AIUM practice guideline for the performance of fetal echocardiography. J Ultrasound Med 2013;32:1067-82.

6. Persico N, Moratalla J, Lombardi CM, et al. Fetal echocardiography at 11-13 weeks by transabdominal high-frequency ultrasound. Ultrasound Obstet Gynecol 2011;37:296-301.

7. De Robertis V, Rembouskos G, Fanelli T, et al. The threevessel and trachea view ( $3 \mathrm{VTV}$ ) in the first trimester of pregnancy: an additional tool in screening for congenital heart defects (CHD) in an unselected population. Prenat Diagn 2017;37:693-8.

8. Pasternok M, Nocun A, Knafel A, et al. "Y Sign" at the Level of the 3-Vessel and Trachea View: An Effective Fetal Marker of Aortic Dextroposition Anomalies in the First Trimester. J Ultrasound Med 2018;37:1869-80.

9. Haak MC, Twisk JW, Van Vugt JM. How successful is fetal echocardiographic examination in the first trimester of pregnancy?. Ultrasound Obstet Gynecol 2002;20:9-13.

10. Salomon LJ, Alfirevic Z, Bilardo CM, et al. ISUOG practice guidelines: performance of first-trimester fetal ultrasound scan. Ultrasound Obstet Gynecol 2013;41:10213. Erratum in: Ultrasound Obstet Gynecol 2013;41:240.

11. Yu D, Sui L, Zhang N. Performance of First-Trimester Fetal Echocardiography in Diagnosing Fetal Heart Defects: Meta-analysis and Systematic Review. J Ultrasound Med 2020;39:471-80.

12. Comas Gabriel C, Galindo A, Martínez JM, et al. Early prenatal diagnosis of major cardiac anomalies in a highrisk population. Prenat Diagn 2002;22:586-93.

13. Sim J, Wright CC. The kappa statistic in reliability studies: use, interpretation, and sample size requirements. Phys Ther 2005;85:257-68.

14. Hernandez-Andrade E, Patwardhan M, Cruz-Lemini M, et al. Early Evaluation of the Fetal Heart. Fetal Diagn Ther 2017;42:161-73.

15. Tegnander E, Eik-Nes SH. The examiner's ultrasound experience has a significant impact on the detection rate of congenital heart defects at the second-trimester fetal examination. Ultrasound Obstet Gynecol 2006;28:8-14.

16. Hutchinson D, McBrien A, Howley L, et al. FirstTrimester Fetal Echocardiography: Identification of Cardiac Structures for Screening from 6 to 13 Weeks' Gestational Age. J Am Soc Echocardiogr 2017;30:763-72.

17. Monni G, Pagani G, Stagnati V, et al. How to perform transabdominal chorionic villus sampling: a practical guideline. J Matern Fetal Neonatal Med 2016;29:1499-505.

18. Daugirdaitė V, van den Akker O, Purewal S. Posttraumatic stress and posttraumatic stress disorder after termination of pregnancy and reproductive loss: a systematic review. J Pregnancy 2015;2015:646345.

19. Crowe L, Graham RH, Robson SC, et al. A survey of health professionals' views on acceptable gestational age and termination of pregnancy for fetal anomaly. Eur J Med Genet 2018;61:493-8.

20. Jicinska H, Vlasin P, Jicinsky M, et al. Does FirstTrimester Screening Modify the Natural History of Congenital Heart Disease? Analysis of Outcome of Regional Cardiac Screening at 2 Different Time Periods. Circulation 2017;135:1045-55.

21. Karadzov Orlic N, Egic A, Damnjanovic-Pazin B, et al. Screening performance of congenital heart defects in first trimester using simple cardiac scan, nuchal translucency, abnormal ductus venosus blood flow and tricuspid regurgitation. Congenit Heart Dis 2019;14:1094-101.

22. Turan S, Turan OM, Desai A, et al. First-trimester fetal cardiac examination using spatiotemporal image correlation, tomographic ultrasound and color Doppler imaging for the diagnosis of complex congenital heart disease in high-risk patients. Ultrasound Obstet Gynecol 2014;44:562-7.

23. Quarello E, Lafouge A, Fries N, et al. Basic heart examination: feasibility study of first-trimester systematic simplified fetal echocardiography. Ultrasound Obstet Gynecol 2017;49:224-30. 
24. Smrcek JM, Berg C, Geipel A, et al. Early fetal echocardiography: heart biometry and visualization of cardiac structures between 10 and 15 weeks' gestation. J Ultrasound Med 2006;25:173-82.

25. Zidere V, Bellsham-Revell H, Persico N, et al.

Comparison of echocardiographic findings in fetuses at less than 15 weeks' gestation with later cardiac evaluation. Ultrasound Obstet Gynecol 2013;42:679-86.

26. De Robertis V, Persico N, Volpe G, et al. Tetralogy of Fallot and Outlet Ventricular Septal Defect with Anterior Malalignment Detected at Early Fetal Echocardiography.
Fetal Diagn Ther 2020;47:1-7.

27. Wu LH, Wang N, Xie HN, et al. Cardiovascular Z-scores in fetuses with tetralogy of Fallot. Ultrasound Obstet Gynecol 2014;44:674-81.

28. Salvesen KÅ, Lees C, Abramowicz J, et al. Safe use of Doppler ultrasound during the 11 to $13+6$-week scan: is it possible? Ultrasound Obstet Gynecol 2011;37:625-8.

29. Nemescu D, Berescu A, Onofriescu M, et al. Safety Indices during Fetal Echocardiography at the Time of First-Trimester Scan Are Machine Dependent. PLoS One 2015;10:e0127570.

Cite this article as: Ye B, Wu Y, Chen J, Yang Y, Niu J, Wang H, Wang Y, Cheng W. The diagnostic value of the early extended fetal heart examination at 13 to 14 weeks gestational age in a high-risk population. Transl Pediatr 2021;10(11):2907-2920. doi: 10.21037/tp-21-255 